\title{
Scale Space for Central Catadioptric Systems: Towards a Generic Camera Feature Extractor*
}

\author{
Luis Puig J. J. Guerrero \\ Departamento de Informática e Ingeniería de Sistemas \\ Instituto de Investigación en Ingeniería de Aragón, I3A Universidad de Zaragoza, Spain \\ \{lpuig, jguerrer\}@unizar.es
}

\begin{abstract}
In this paper we propose a new approach to compute the scale space of any omnidirectional image acquired with a central catadioptric system. When these cameras are central they are explained using the sphere camera model, which unifies in a single model, conventional, paracatadioptric and hypercatadioptric systems. Scale space is essential in the detection and matching of interest points, in particular scale invariant points based on Laplacian of Gaussians, like the well known SIFT. We combine the sphere camera model and the partial differential equations framework on manifolds, to compute the Laplace-Beltrami (LB) operator which is a second order differential operator required to perform the Gaussian smoothing on catadioptric images. We perform experiments with synthetic and real images to validate the generalization of our approach to any central catadioptric system.
\end{abstract}

\section{Introduction}

In recent years the interest in omnidirectional cameras has increased considerably. In particular in catadioptric systems which are a combination of lenses and mirrors. Baker and Nayar [2] have shown which catadioptric devices have a single effective viewpoint, i.e. are central cameras. This central property allows to easily calculate the directions of the light rays coming into the camera. The most useful central catadioptric systems are the paracatadioptric and the hypercatadioptric models, using a mirror of paraboloidal/hyperboloidal shape, coupled with an orthographic/perspective camera.

Image processing has developed through the years techniques for conventional (perspective) cameras. Among all these techniques, feature detection/extraction is one of the most relevant, since it represents a crucial step on higher level techniques, such as matching, SLAM, structure from

\footnotetext{
${ }^{*}$ This work has been supported by project DPI2009-14664-C02-01.
}

motion, navigation, localization, surveillance and many more. A particular useful property for features is to be scale-invariant. There are different approaches to detect scale-invariant features. Some of them are based on the scale-space analysis $[15,17]$. Some others are based on the grey scale intensity [13, 16]. SIFT [15] has become the most used feature extraction approach. It has also been used directly in omnidirectional images [10], although it is not designed to work on them. This SIFT approach has inspired different works trying to replicate its good results on different imagery systems, in particular on wide-angle cameras. In [6] a Gaussian kernel is derived. It requires the omnidirectional image to be mapped to the sphere. Then, the spherical Fourier transform is computed and convolved with the spherical Gaussian function. In [12] the image is mapped to the sphere and obtain scale-space images as the solution to the heat diffusion equation on the sphere which is implemented in the frequency domain using spherical harmonics. This approach introduces new drawbacks as aliasing and bandwidth selection. A complete SIFT version computed on the sphere also using the heat diffusion equation is presented in [7]. In [11] an approximation to spherical diffusion using stereographic projection is proposed. It maps the omnidirectional image to the stereographic image plane through the sphere. It also maps the spherical Gaussian function to an equivalent kernel on the stereographic image plane. Then, the approximate spherical diffusion is defined as the convolution of the stereographic image with the stereographic version of the Gaussian kernel. More recently [14] propose an improvement to the SIFT detector by introducing radial distortion into the scale-space computation. In [5] a framework to perform scale space analysis for omnidirectional images using partial differential equations is proposed. It leads to the implementation of the linear heat flow on manifolds through the Laplace-Beltrami operator. Omnidirectional images are treated as scalar fields on parametric manifolds. Based on this work Arican and Frossard [1] propose a scale invariant feature detector for omnidirectional images. They deal with the paracatadioptric projec- 


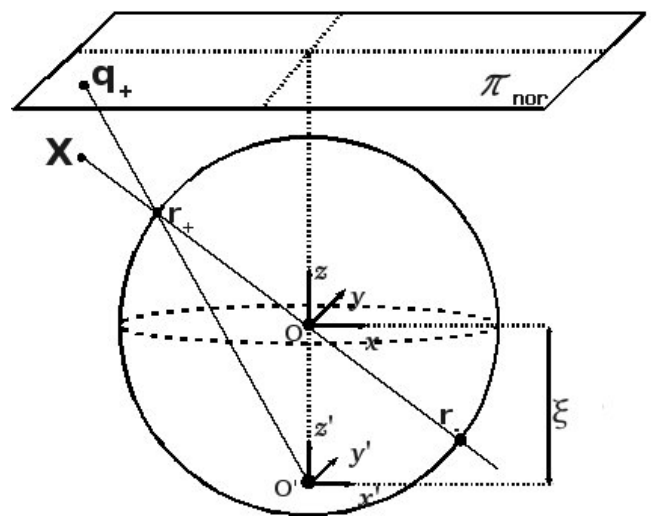

Figure 1. Sphere Camera Projection Model

tion which is equivalent to the inverse of the stereographic projection. They model this projection on the sphere and obtain its corresponding metric. This metric is conformallly equivalent to the Euclidean one making the computation of the Laplace-Beltrami operator straightforward. Although this approach could work with any catadioptric system, the metric describing the reflecting surface (mirror) has to be provided, which in some cases can be difficult to obtain.

In this paper we propose a new approach to compute the scale-space for any central catadioptric system. We integrate the sphere camera model $[9,3]$, which describes any central catadioptric system, selecting it by one single parameter, with the partial differential equations on manifolds framework through the heat diffusion equation [5, 1]. Using this framework and the mirror parameter we compute the metric representing that particular reflecting surface. Then we use this metric to calculate the corresponding LaplaceBeltrami operator. This second order operator allow us to perform the Gaussian smoothing on omnidirectional images.

The rest of the paper is organized as follows. In section 2 we explain the sphere camera model and its geometrical interpretation for the different catadioptric systems. In section 3 the computation of the Laplace-Beltrami operator considering a generic catadioptric system is developed. Section 4 show experiments computing the scale space for different catadioptric systems using synthetic and real images. Finally in section 5 we summarize the results of this paper.

\section{Sphere Camera Model}

According to [9, 3], all central catadioptric cameras can be modeled by a unit sphere and a perspective projection, such that the projection of 3D points can be performed in two steps (Fig. 1). First, one projects the point onto the unit sphere, obtaining the intersection of the sphere and the line joining its center and the 3D point. There are two such intersection points, which are represented as $\mathbf{r}_{ \pm}$. These points are then projected in the second step, using a per- spective projection $M$ resulting in two image points, $\mathbf{q}_{ \pm}$, one of which is physically true. This model covers all central catadioptric cameras, encoded by $\xi$, which is the distance between the perspective projection and the center of the sphere. We have $\xi=0$ for perspective, $\xi=1$ for paracatadioptric and $0<\xi<1$ for hypercatadioptric systems.

A point on the sphere $\mathbf{r}=(X, Y, Z)$ can also be represented by two angles $(\theta, \varphi)$ (Fig. 2a). Depending on the location of the projection point (mirror parameter $\xi$ ) we can have different radial projection functions [8]. In the paracatadioptric projection case $(\xi=1)$, which coincides with the inverse stereographic projection (Fig. 2c), its radial projection function is $2 \tan \left(\frac{\theta}{2}\right)$. In the perspective case where $\xi=0$ the corresponding projection function is $\tan (\theta)$. In the hypercatadioptric case $(0<\xi<1)$ the radial projection function becomes $(1+\xi) \frac{\sin \theta}{\xi-\cos \theta}$ (Fig. 2d). This function will be useful later to generalize the computing of the metric for any mirror shape in central catadioptric systems.

\section{Scale Space of Catadioptric Images}

In this section we integrate the sphere camera model (section 2) that models any central catadioptric system and the techniques developed to compute the differential operators on non-Euclidean manifolds [4] such as the mirror surfaces present in catadioptric systems.

\subsection{Differential Operators on Riemannian Mani- folds}

The scale space representation $I(x, y, t)$ is computed using the heat diffusion equation and differential operators on the non-Euclidean manifolds. It is defined as

$$
\frac{\partial I(x, y, t)}{\partial t}=\Delta I(x, y, t)
$$

where $\Delta$ is the Laplacian-Beltrami operator and $t$ is the scale level. The initial condition is $I\left(x, y, t_{0}\right)=I(x, y)$ where $I(x, y)$ is the original image.

We briefly define the differential operators on the manifolds which make possible the computation of the LaplaceBeltrami operator. Let $\mathcal{M}$ be a parametric surface on $\mathbb{R}^{3}$ with an induced Riemannian metric $g_{i j}$ that encodes the geometrical properties of the manifold.

In a local system of coordinates $x^{i}$ on $\mathcal{M}$, the components of the gradient reads

$$
\nabla^{i}=g^{i j} \frac{\partial}{\partial x^{j}}
$$

where $g^{i j}$ is the inverse of $g_{i j}$. A similar reasoning is used to obtain the expression of the divergence of a vector field X on $\mathcal{M}$

$$
\operatorname{div} \mathbf{X}=\frac{1}{\sqrt{g}} \partial_{i}\left(\mathbf{X}^{i} \sqrt{g}\right)
$$




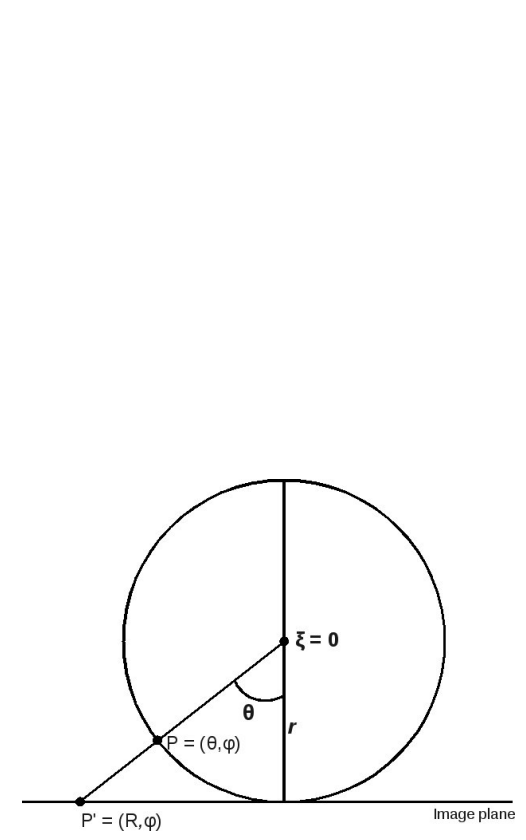

(b)

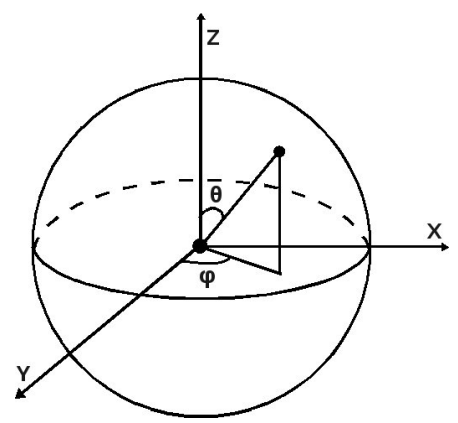

(a)

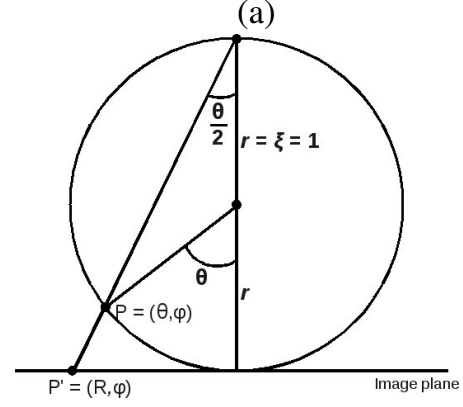

(c)

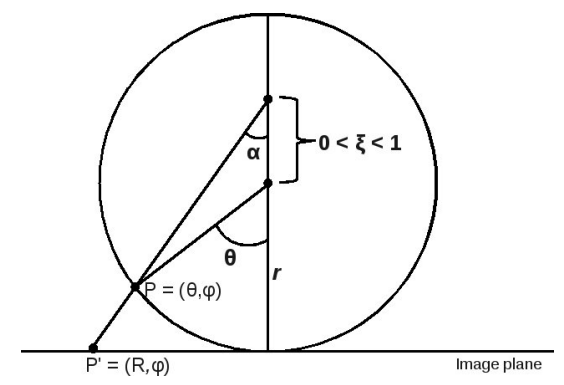

(d)

Figure 2. Simplified sphere camera model, showing the different radial projection functions. (a) spherical coordinates. (b) perspective projection. (c) paracatadioptric projection. (d) hypercatadioptric projection.

where $g$ is the determinant of $g^{i j}$. Finally, combining these two operators we obtain the Laplace-Beltrami operator, which is the second order differential operator defined on scalar fields on $\mathcal{M}$ by

$$
\Delta I=\frac{1}{\sqrt{g}} \partial_{j}\left(\sqrt{g} g^{i j} \partial_{i} I\right)
$$

\subsection{Computing a Generic Metric on the Sphere}

As explained in section 2 omnidirectional images are formed in two steps. The first one projects a 3D point to the unitary sphere. Then this point is projected from the unitary sphere to the image plane through a variable projection point, which is determined by the geometry of the mirror (parameter $\xi$ ). If the system is calibrated [18], it is also possible like in any conventional camera, to map the catadioptric image to the unitary sphere.

In $[5,1]$, the mapping from paracatadioptric images to the sphere is used for the computation of the differential operators explained before. This allows to process the spherical image directly using the image coordinates. In this paper we extend that approach to all central catadioptric systems.

Consider the unitary sphere $\mathbb{S}^{2}$ (Fig. 2a). A point on $\mathbb{S}^{2}$ can be represented in cartesian and polar coordinates as

$$
(\mathrm{X}, \mathrm{Y}, \mathrm{Z})=(\sin \theta \cos \varphi, \sin \theta \sin \varphi, \cos \theta)
$$

The Euclidean element in cartesian and polar coordinates is defined as

$$
\mathrm{d} l^{2}=\mathrm{dX} X^{2}+\mathrm{d} \mathrm{Y}^{2}+\mathrm{dZ} Z^{2}=\mathrm{d} \theta^{2}+\sin ^{2} \theta \mathrm{d} \varphi^{2}
$$

Under the sphere camera model, a point on the sphere $(\theta, \varphi)$, is mapped to a point in polar coordinates $(R, \varphi)$ in the image plane. The $\theta$ angle depends on the central catadioptric system we are dealing with, while $\varphi$ remain the same. For example, a conventional perspective system is described with $\theta=\arctan (R)$ and a paracatadioptric system with $\theta=2 \arctan \left(\frac{R}{2}\right)$. In the general case (see Fig. 2d) we have

$$
\theta=\arctan \left(\frac{R\left(1+\xi+\sqrt{(1+\xi)^{2}-R^{2}\left(\xi^{2}-1\right)}\right)}{1+\xi-R^{2} \xi+\sqrt{(1+\xi)^{2}-R^{2}\left(\xi^{2}-1\right)}}\right)
$$

In terms of these new coordinates the metric becomes

$\mathrm{d} l^{2}=\frac{\left(\xi+\xi^{2}+\sqrt{(1+\xi)^{2}-R^{2}\left(\xi^{2}-1\right)}\right)^{2}\left(R^{2} \mathrm{~d} \varphi^{2}+\frac{(1+\xi) \mathrm{d} R^{2}}{1-R^{2}(\xi-1)+\xi}\right)}{\left(R^{2}+(1+\xi)^{2}\right)^{2}}$

Let $(x, y) \in \mathbb{R}^{2}$ on the sensor plane define cartesian coordinates, where $R^{2}=x^{2}+y^{2}$ and $\varphi=\arctan \left(\frac{y}{x}\right)$

$$
\mathrm{d} l^{2}=\lambda\left(4(y \mathrm{~d} x-x \mathrm{~d} y)^{2}-\frac{4(1+\xi)(x \mathrm{~d} x+y \mathrm{~d} y)^{2}}{\left(x^{2}+y^{2}\right)(\xi-1)-\xi-1}\right)
$$

where

$$
\lambda=\frac{\left(\xi+\xi^{2}+\sqrt{(1+\xi)^{2}-\left(x^{2}+y^{2}\right)\left(\xi^{2}-1\right)}\right)^{2}}{4\left(x^{2}+y^{2}\right)\left(x^{2}+y^{2}+(1+\xi)^{2}\right)^{2}}
$$


To verify the correctness of this approach we substitute $\xi$ to the values for which we already know the metric. For $\xi=1$ the result is the same as that one presented in [1]. In the case of $\xi=0$ that corresponds to the perspective case we expect a cartesian metric but what we get is the following

$$
\mathrm{d} l^{2}=\frac{\left.\left(1+y^{2}\right) \mathrm{d} x^{2}-2 x y \mathrm{~d} x \mathrm{~d} y+\left(1+x^{2}\right) \mathrm{d} y^{2}\right)}{\left(1+x^{2}+y^{2}\right)^{2}}
$$

This is explained since there is no map from the sphere to the plane being both conformal and area-preserving. However, from the generic metric we have already computed, we are able to compute the Laplace-Beltrami operator. To deal with the perspective case we only need to use the classical Laplacian.

\subsection{Computing the Generic Metric on the Normal- ized Plane}

The previous approach computes the metric on the sphere and then it is projected to the image plane through the angle $\theta$ which encodes the radial projection function. We have observed that using the sphere camera model we are able to compute the metric directly on the image plane and at the same time take into account the geometry of the mirror, which is given by the mirror parameter $\xi$.

Let's observe the perspective projection in cartesian and spherical coordinates

$$
\left(\frac{\mathrm{X}}{\mathrm{Z}}, \frac{\mathrm{Y}}{\mathrm{Z}}\right)=(\tan \theta \cos \varphi, \tan \theta \sin \varphi)=(x, y)
$$

The radial component is $R=\sqrt{x^{2}+y^{2}}=\tan (\theta)$ and the Euclidean element in this case just takes into account the $(x, y)$ coordinates but also the two spherical coordinates $(\theta, \varphi)$

$$
\mathrm{d} s^{2}=\mathrm{d}(\tan \theta \cos \varphi)^{2}+\mathrm{d}(\tan \theta \sin \varphi)^{2}=\mathrm{d} x^{2}+\mathrm{d} y^{2}
$$

The same approach applied to the paracatadioptric case $\xi=1$ gives

$$
\left(\frac{\mathrm{X}}{1-\mathrm{Z}}, \frac{\mathrm{Y}}{1-\mathrm{Z}}\right)=\left(\frac{\sin \theta \cos \varphi}{1-\cos \theta}, \frac{\cos \theta \cos \varphi}{1-\cos \theta}\right)=(x, y)
$$

and its corresponding Euclidean element with $\theta=$ $2 \arctan \left(\frac{\sqrt{x^{2}+y^{2}}}{2}\right)$ and $\varphi=\arctan \left(\frac{y}{x}\right)$ becomes

$$
\mathrm{d} l^{2}=\frac{16\left(\mathrm{~d} x^{2}+\mathrm{d} y^{2}\right)}{\left(x^{2}+y^{2}-4\right)^{2}\left(\sqrt{\frac{\left(x^{2}+y^{2}+4\right)^{2}}{\left(x^{2}+y^{2}-4\right)^{2}}}-1\right)^{2}}
$$

The generic metric equation for any catadioptric system is described by the following Euclidean element on the normalized plane

$$
\mathrm{d} s^{2}=\frac{(\xi \cos \theta-1)^{2} \mathrm{~d} \theta^{2}+(\xi-\cos \theta)^{2} \sin ^{2} \theta \mathrm{d} \varphi^{2}}{(\xi-\cos \theta)^{4}}
$$

which allows to compute the Euclidean element on the normalized plane with the substitution of $\theta$ and $\varphi$ in terms of $x$ and $y$ (that equation is not shown here because of lack of space).

\subsection{Generic Laplace-Beltrami Operator}

From (9) we can compute the generic metric in matrix form $g_{i j}$ and its corresponding inverse matrix $g^{i j}$

$$
g^{i j}=\gamma_{2}\left(\begin{array}{cc}
-x^{2}(\xi-1)+\xi+1 & x y(\xi-1) \\
x y(\xi-1) & -y^{2}(\xi-1)+\xi+1
\end{array}\right)
$$

with

$$
\gamma_{2}=\frac{\left(x^{2}+y^{2}+(1+\xi)^{2}\right)^{2}}{(1+\xi)\left(\xi+\xi^{2}+\sqrt{1-\left(x^{2}+y^{2}\right)\left(\xi^{2}-1\right)+2 \xi+\xi^{2}}\right)^{2}}
$$

The determinant of (16) is

$$
\operatorname{det}\left(g^{i j}\right)=-\frac{\left(x^{2}+(1+\xi)^{2}+y^{2}\right)^{4}\left(\left(x^{2}+y^{2}\right)(\xi-1)-\xi-1\right)}{(1+\xi)\left(\xi+\xi^{2}+\sqrt{1-\left(x^{2}+y^{2}\right)\left(\xi^{2}-1\right)+2 \xi+\xi^{2}}\right)^{4}}
$$

With all these elements we are now able to compute the Laplace-Beltrami operator (4) which is represented by the differential operators

$$
\begin{gathered}
\Delta I=\lambda_{1} \frac{\partial^{2} I}{\partial x^{2}}+\lambda_{2} \frac{\partial^{2} I}{\partial y^{2}}+\lambda_{3} \frac{\partial^{2} I}{\partial x y}+\lambda_{4} \frac{\partial I}{\partial x}+\lambda_{5} \frac{\partial I}{\partial y} \\
\lambda_{1}=\frac{\left(x^{2}\left(\xi^{2}-1\right)-1\right)\left(1+x^{2}+y^{2}\right)^{2}}{-1-\xi^{2}+\left(\xi^{2}-1\right)\left(x^{2}+y^{2}\right)-2 \xi \sqrt{1-\left(\xi^{2}-1\right)\left(x^{2}+y^{2}\right)}}
\end{gathered}
$$

$$
\lambda_{2}=\frac{\left(y^{2}\left(\xi^{2}-1\right)-1\right)\left(1+x^{2}+y^{2}\right)^{2}}{-1-\xi^{2}+\left(\xi^{2}-1\right)\left(x^{2}+y^{2}\right)-2 \xi \sqrt{1-\left(\xi^{2}-1\right)\left(x^{2}+y^{2}\right)}}
$$

$$
\lambda_{3}=\frac{2 x y\left(\xi^{2}-1\right)\left(1+x^{2}+y^{2}\right)^{2}}{-1-\xi^{2}+\left(\xi^{2}-1\right)\left(x^{2}+y^{2}\right)-2 \xi \sqrt{1-\left(\xi^{2}-1\right)\left(x^{2}+y^{2}\right)}}
$$

$$
\lambda_{4}=\frac{4 x\left(1+x^{2}+y^{2}\right)\left(2-\xi^{2}-2\left(\xi^{2}-1\right)\left(x^{2}+y^{2}\right)-\xi \sqrt{1-\left(\xi^{2}-1\right)\left(x^{2}+y^{2}\right)}\right)}{-1-\xi^{2}+\left(\xi^{2}-1\right)\left(x^{2}+y^{2}\right)-2 \xi \sqrt{1-\left(\xi^{2}-1\right)\left(x^{2}+y^{2}\right)}}
$$


$\lambda_{5}=\frac{4 y\left(1+x^{2}+y^{2}\right)\left(2-\xi^{2}-2\left(\xi^{2}-1\right)\left(x^{2}+y^{2}\right)-\xi \sqrt{1-\left(\xi^{2}-1\right)\left(x^{2}+y^{2}\right)}\right)}{-1-\xi^{2}+\left(\xi^{2}-1\right)\left(x^{2}+y^{2}\right)-2 \xi \sqrt{1-\left(\xi^{2}-1\right)\left(x^{2}+y^{2}\right)}}$

An analogous process has been performed for the computation of the metric in the normalized plane, but the equations are too big to fit in these pages. In the rest of the paper we use the metric computed on the sphere.

\subsection{Smoothing Catadioptric Images Using the Generic Laplace-Beltrami Operator}

We compute the smoothing of the catadioptric images using the heat diffusion equation. This equation is computed at successive time steps, $t_{i}=k^{2 i} \sigma_{o}^{2}$ is defined in terms of the normalization and scale factors $k$ and the base smoothing level $\sigma_{o}$.

The differentiation with respect to time in the heat diffusion equation is discretized with time intervals, $d_{t}$. To compute the discrete differential representations of the image $\frac{\partial I}{\partial x}, \frac{\partial I}{\partial y}, \frac{\partial^{2} I}{\partial x y}, \frac{\partial^{2} I}{\partial x^{2}}$ and $\frac{\partial^{2} I}{\partial y^{2}}$ we convolve the catadioptric image with different kernels

$$
\begin{array}{r}
I_{x}=\left[\begin{array}{ll}
-1 & 1
\end{array}\right] \quad I_{y}=\left[\begin{array}{r}
-1 \\
1
\end{array}\right] \quad I_{x x}=\left[\begin{array}{lll}
1 & -2 & 1
\end{array}\right] \\
I_{y y}=\left[\begin{array}{r}
1 \\
-2 \\
1
\end{array}\right] \quad I_{x y}=\left[\begin{array}{rrr}
0 & 1 & 0 \\
1 & -4 & 1 \\
0 & 1 & 0
\end{array}\right]
\end{array}
$$

For a particular catadioptric system defined by $\xi$ we compute the corresponding coefficients (19-23) which multiply their corresponding convolved image. Then we compute the specific Laplace-Beltrami operator. Finally, smoothing is performed by updating $I(x, y, t)$ with the differences that have been computed at previous time steps. We summarize the process in algorithm 1.

\section{Experiments}

In this section we perform several experiments using synthetic and real images. The synthetic catadioptric images are generated using the raytracing software POVray ${ }^{1}$ and correspond to images from an office scene ${ }^{2}$. Two hypercatadioptric systems with mirror parameters of $\xi=$ $0.9662^{3}(\mathrm{~m} 1)$ and $\xi=0.7054^{4}(\mathrm{~m} 2)$ are considered. We also consider one paracatadioptric system $\xi=1$ with radius $r=2.5 \mathrm{~cm}$. The real images are acquired using the hypercatadioptric system designed by Neovision (m1).

\footnotetext{
${ }^{1}$ http://www.povray.org

${ }^{2}$ http://www.ignorancia.org

${ }^{3}$ http://www.neovision.cz

${ }^{4}$ http://www.accowle.com
}
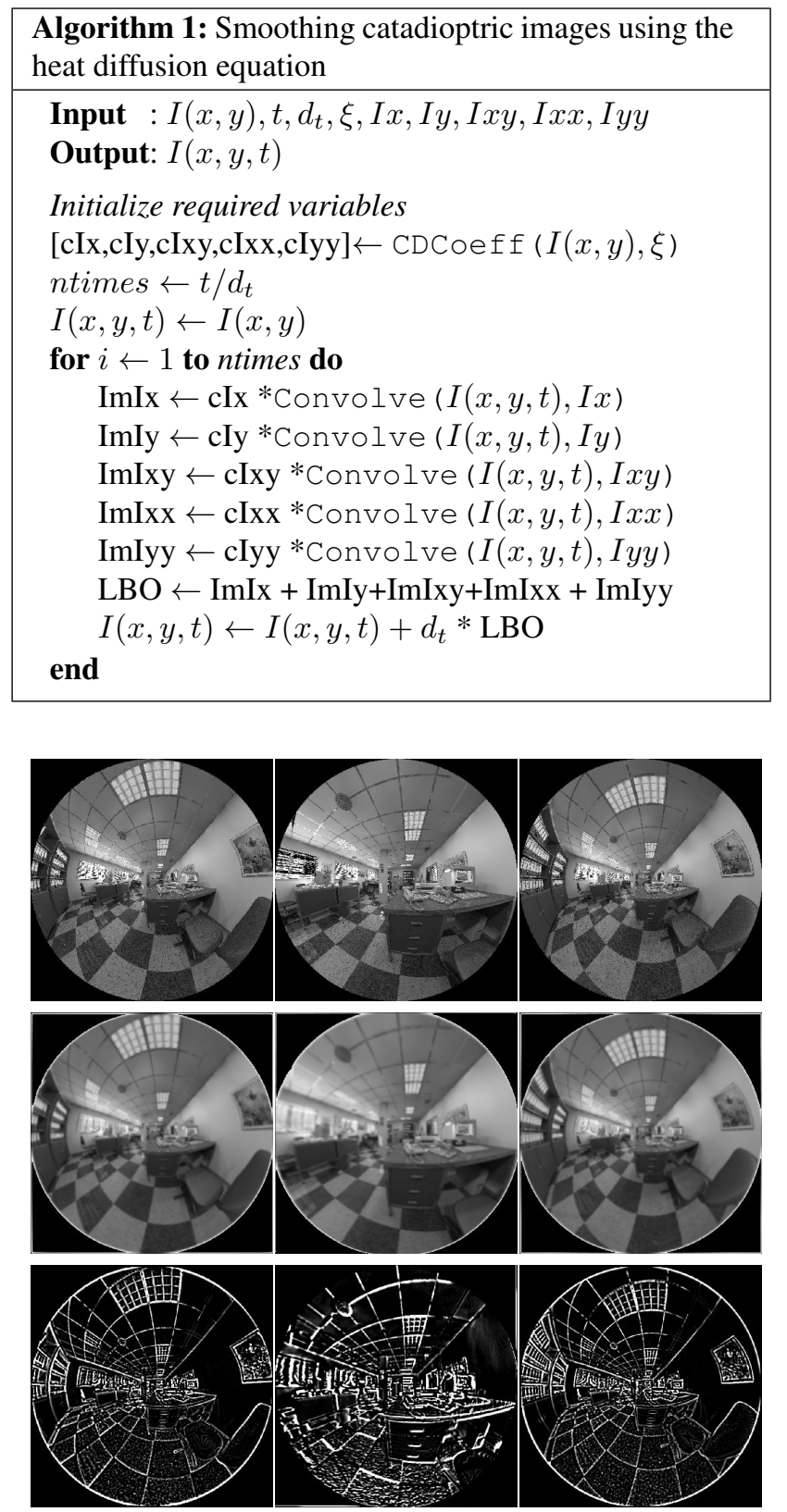

(a)

(b)

(c)

Figure 3. Smoothed catadioptric images with $t=3$. (a) $\mathrm{m} 1$. (b) $\mathrm{m} 2$ and (c) paracatadioptric. The first row represents the original images, the second represents the smoothed images and the last one the corresponding LB operators.

\subsection{Smoothing of Synthetic Images}

We follow the algorithm 1 with $d_{t}=0.01$. All the coefficients $\lambda_{i}, i=1 \ldots 5$ are computed once since they only depend on the geometry of the image and not on the gray values. Fig. 3 shows smoothed images with different mirrors at the same scale factor $t=3$. They also show the corresponding generic LB operator. We observe that the geometry of 

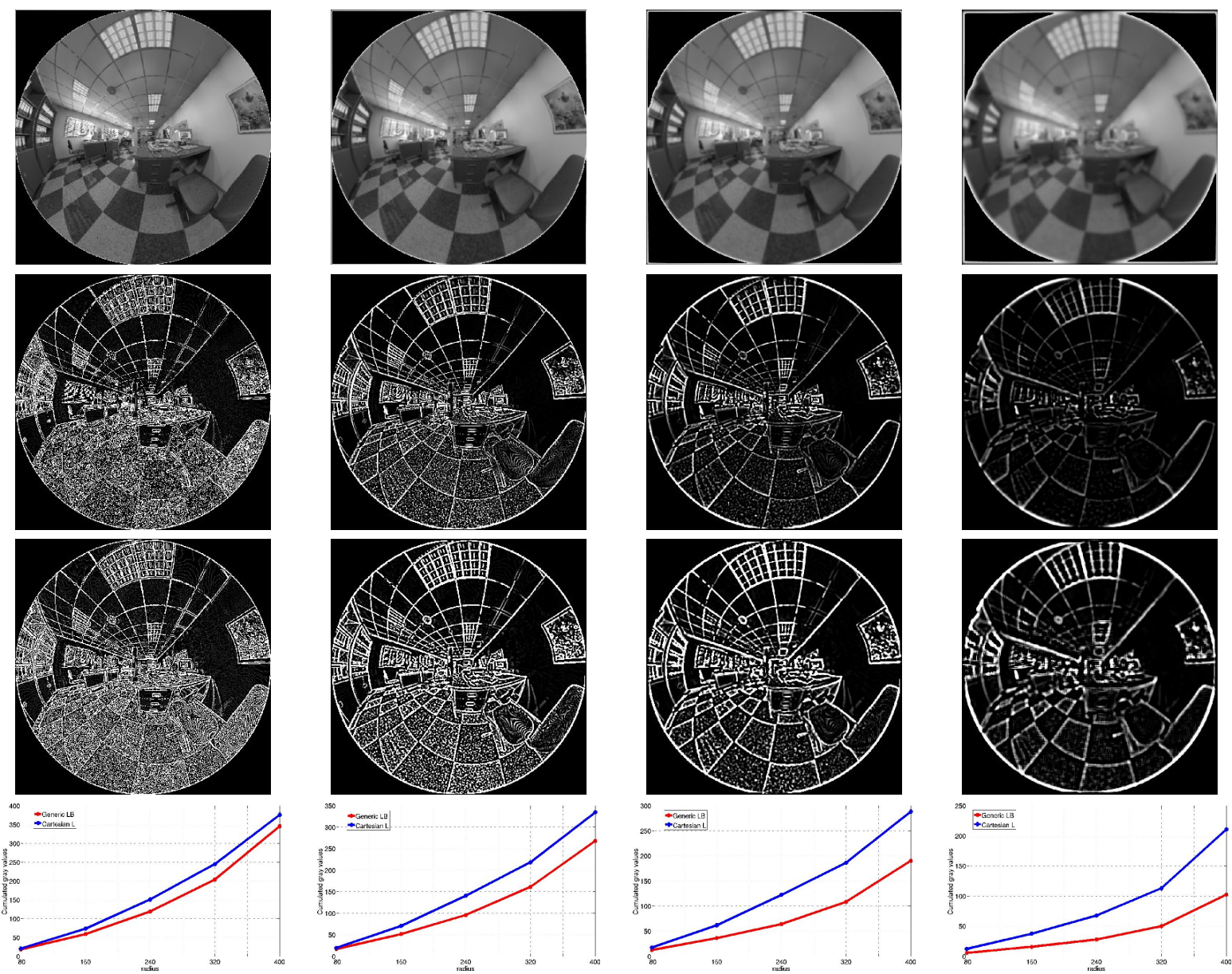

Figure 4. Comparison between generic LB operator and cartesian Laplacian. The first row show the smoothed images for scales $t=$ $\{1,2,3,5\}$ using our approach. The second row shows the corresponding generic LB operators. The third row, the cartesian Laplacians. The last row presents the sum of the values (scaled for visualization purposes) of the generic LB and the cartesian Laplacian as a function of the radius of a circle with origin at the image center.

each mirror is taken into account when the smoothing is performed. The LB operator of $\mathrm{m} 1$ and the one corresponding to the paracatadioptric system are similar since the mirror parameter is close to 1 . In the LB operators of mirrors $\mathrm{m} 1$ and $\mathrm{m} 2$, we observe how the intensities and thickness of the edges vary with respect to the distance to the image center. The differences between these LB operators are explained since the geometries of these mirrors are different.

In order to verify the validity of our approach we perform an experiment where we compare the generic LB operator computed using our approach to the cartesian Laplace operator. To obtain this operator we smooth the omnidirectional image to the same scale $t$, using the corresponding Gaussian kernel. Then, we compute the Laplacian of this image using the cartesian operator $I_{x y}$. We select the hypercatadioptric (m1) image for which we compute scales $t=\{1,2,3,5\}$ and their corresponding generic LB and cartesian Laplacian. In Fig. 4 we show the results. We observe that the generic LB operator considers a difference between the pixels close to the center and those close to the periphery, while the cartesian Laplacian has the same effect on all pixels, without taking into account their location with respect to the center.

To quantify the last statement we sum the values of the generic LB operator inside circles with different radii and origin at the image center. We perform the same procedure with the cartesian Laplacian. The last row of Fig. 4 shows the comparison of these sums for the the different scales. We observe that the pixels in the periphery have smaller values in the generic LB operator than those on the normal Laplace operator. In the center where the resolution of the catadioptric images is bigger the values of the LaplaceBeltrami operator and the normal Laplace are similar.

\subsection{Scale Space on Real Catadioptric Images}

In Fig. 5 we show the pyramid that compose the scale space of a hypercatadioptric image computed using our approach. We define the scale space to be composed of four 


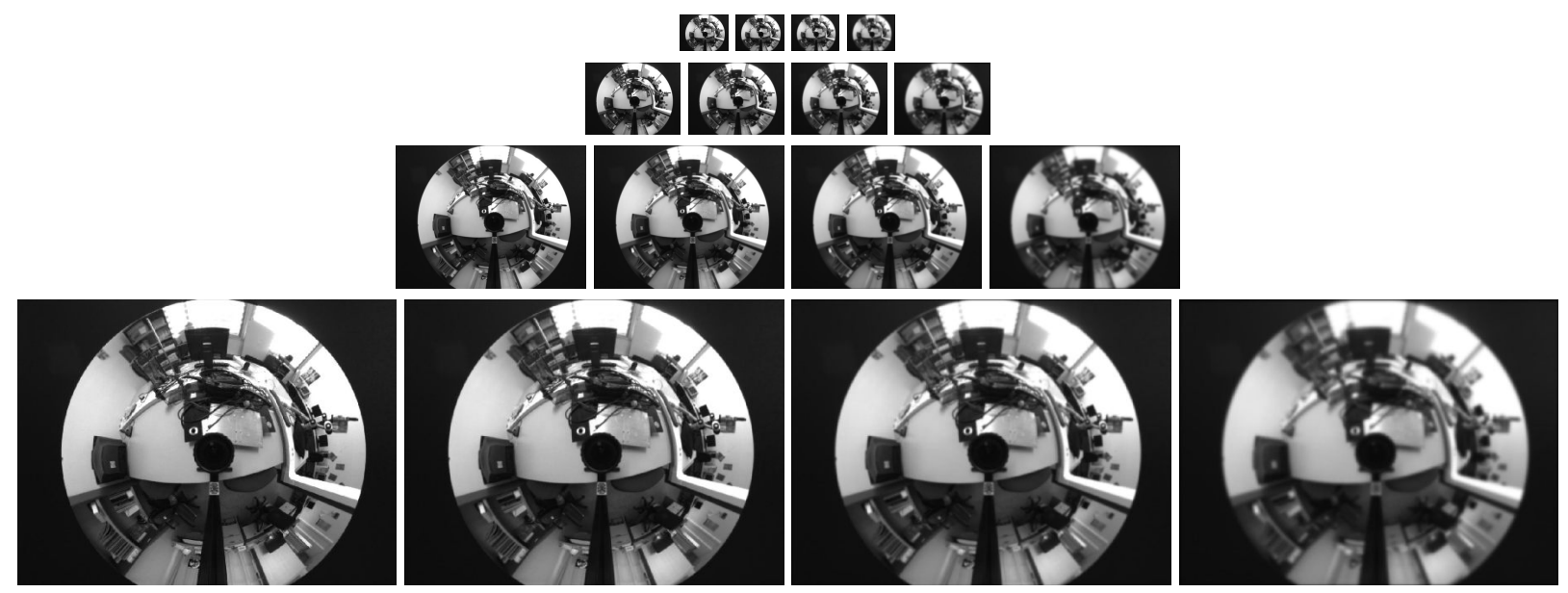

Figure 5. Scale Space of a real omnidirectional image, composed of four octaves and four scales per octave. First octave $1024 \times 768$ pixels. Second octave $512 \times 384$ pixels. Third octave $256 \times 192$ pixels. Fourth octave $128 \times 96$ pixels.

octaves and four scales per octave. The initial sigma is defined as $t=0.8$ and the rest are computed as explained in Section 3.5. The value of the smoothing interval is defined as $k=2^{1 / 3}$.

\subsection{Repeatability Experiment}

In this experiment we test the repeatability of the extrema points detected with the scale space computed using our approach. The extrema points are obtained from the approximation to the scale-normalized Laplacian of Gaussian through differences of Gaussians, similar to [15]. We generate nine synthetic images with rotations from zero degrees to eighty degrees around the $z$-axis, with steps of ten degrees between each pair of images. The two extreme images are shown in Fig. 6. We observe a drastic distortion on the images, this is produced by the conic shape of the mirror.

Since the rotation of the catadioptric system is known, we can map the detected features in the reference image $\overline{\mathbf{x}}$ to the subsequent images and compute the distance between them. The matching criteria is the following

$$
\|\hat{\mathbf{x}}-\overline{\mathbf{x}}\| \leq \delta_{d}
$$

where, $\hat{\mathbf{x}}$ is the mapped point. We use the Euclidean distance. The distance threshold $\delta_{d}$ has to be adapted to capture the matches with different scale levels and computed as

$$
\delta_{d}=\delta_{0} \cdot t_{\overline{\mathbf{x}}},
$$

where $\delta_{0}$ is the distance threshold parameter.

We compare our approach to SIFT algorithm. In particular we use Vedaldi's implementation [19]. In Fig. 7 we show the results of this experiment. We observe that the LB approach has a clear advantage over the classical SIFT approach, obtaining double repeatability than the scale space

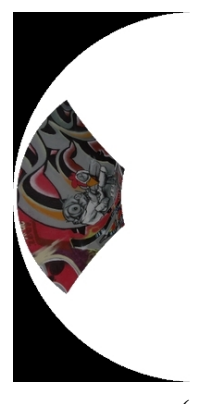

(a)

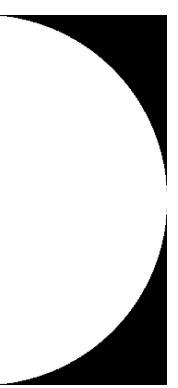

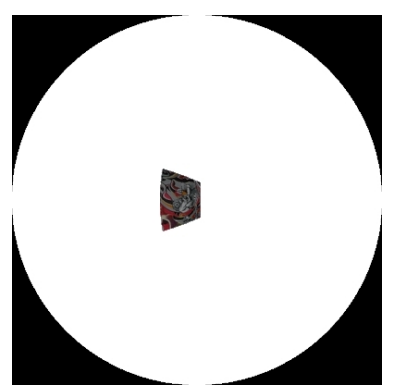

(b)
Figure 6. Synthetic hypercatadioptric images. (a) Reference image. (b) Image generated with the catadioptric system rotated $80^{\circ}$ around the $z$-axis.

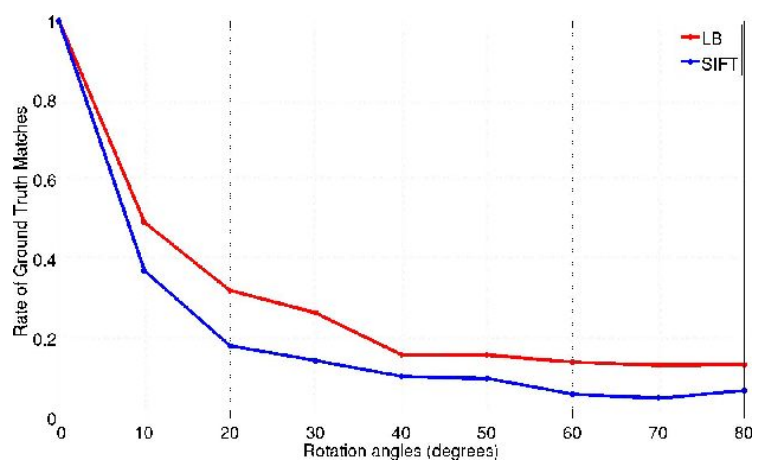

Figure 7. Percentage of correct matches through rotations of the catadioptric system around the $z$-axis. The $x$-axis represents the orientation of the catadioptric system in degrees with respect to the initial position.

used by SIFT in extreme camera rotations. The low rate shows the difficulties of matching catadioptric images. 


\section{Conclusions and Future Work}

We have presented a new way to compute the scale-space of omnidirectional images. We integrate the sphere camera model which considers all central catadioptric systems with the partial differential equation framework on manifolds to compute a generic version of the second order differential operator Laplace-Beltrami. This operator is used to perform the Gaussian smoothing on catadioptric images. We perform experiments using synthetic images generated with parameters coming from actual manufactured mirrors. We observe that Laplace-Beltrami operator considers correctly the geometry of the mirror, since the pixels at the periphery have a different weight than those at the center. This situation explains the natural non-homogeneous resolution inherent to the central catadioptric systems. The near future work is to implement a complete scale-invariant feature detector also invariant to camera since the sphere camera model allows to consider all central projections.

\section{References}

[1] Z. Arican and P. Frossard. OmniSIFT: Scale Invariant Features in Omnidirectional Images. In Proceedings of the International Conference on Image Processing (ICIP), 2010. $1,2,3,4$

[2] S. Baker and S. Nayar. Single viewpoint catadioptric cameras, 2001. 1

[3] J. P. Barreto and H. Araujo. Issues on the geometry of central catadioptric image formation. Proceedings of the Conference on Computer Vision and Pattern Recognition, 2:422427, 2001. 2

[4] M. Bertalmío, L.-T. Cheng, S. Osher, and G. Sapiro. Variational problems and partial differential equations on implicit surfaces. Journal of Computational Physics, 174(2):759 780, 2001. 2

[5] I. Bogdanova, X. Bresson, J.-P. Thiran, and P. Vandergheynst. Scale space analysis and active contours for omnidirectional images. IEEE Transactions on Image Processing, 16(7):1888-1901, july 2007. 1, 2, 3

[6] T. Bulow. Spherical diffusion for $3 \mathrm{~d}$ surface smoothing. IEEE Transactions on Pattern Analysis and Machine Intelligence, 26(12):1650-1654, dec. 2004. 1

[7] J. Cruz, I. Bogdanova, B. Paquier, M. Bierlaire, and J.-P. Thiran. Scale Invariant Feature Transform on the Sphere: Theory and Applications. Technical report, EPFL, 2009. 1

[8] M. Fleck. Perspective projection: The wrong imaging model. Technical report, Univ. of Iowa, 1995. 2

[9] C. Geyer and K. Daniilidis. A unifying theory for central panoramic systems and practical applications. In Proceedings of the 6th European Conference on Computer VisionPart II, pages 445-461, London, UK, 2000. 2

[10] J. J. Guerrero, A. C. Murillo, and C. Sagues. Localization and matching using the planar trifocal tensor with bearingonly data. IEEE Transactions on Robotics, 24(2):pp. 494501, 2008. 1
[11] P. Hansen, P. Corke, and W. Boles. Wide-angle visual feature matching for outdoor localization. The International Journal of Robotics Research, 29:267-297, 2010. 1

[12] P. Hansen, P. Corke, W. Boles, and K. Daniilidis. Scaleinvariant features on the sphere. In IEEE 11th International Conference on Computer Vision, pages 1-8, 2007. 1

[13] T. Kadir and M. Brady. Saliency, scale and image description. Int. J. Comput. Vision, 45:83-105, 2001. 1

[14] M. Lourenço, J. Barreto, and A. Malti. Feature detection and matching in images with radial distortion. In IEEE International Conference on Robotics and Automation, pages 1028 $-1034,2010.1$

[15] D. Lowe. Distinctive image features from scale-invariant keypoints. In International Journal of Computer Vision, volume 20, pages 91-110, 2004. 1, 7

[16] J. Matas, O. Chum, M. Urban, and T. Pajdla. Robust wide baseline stereo from maximally stable extremal regions. In British Machine Vision Conference, 2002. 1

[17] K. Mikolajczyk and C. Schmid. Scale \& affine invariant interest point detectors. Int. J. Comput. Vision, 60:63-86, October 2004. 1

[18] L. Puig, Y. Bastanlar, P. Sturm, J. J. Guerrero, and J. Barreto. Calibration of central catadioptric cameras using a dlt-like approach. Int. J. Comput. Vision, 93:101-114, 2011. 3

[19] A. Vedaldi. An open implementation of SIFT detector and descriptor. Technical Report 070012, UCLA CSD, 2007. 7 